\title{
Business Intelligence for Business Intelligence: A case study at STMicroelectronics
}

\author{
Manel Brichni ${ }^{1,2,3}$, Sophie Dupuy-Chessa ${ }^{1}$, Lilia Gzara $^{2}$, Nadine Mandran $^{1}$, Corinne Jeannet ${ }^{3}$ \\ ${ }^{1}$ Univ. Grenoble Alpes, LIG, F-38000 Grenoble, France \\ CNRS, UPMF, F-38000 Grenoble, France \\ \{manel.brichni,sophie.dupuy,nadine.mandran\}@imag.fr \\ CNRS, UPMF, F-38000 Grenoble, Franc ${ }^{2}$ Univ. Grenoble Alpes, G-SCOP, F-38000 Grenoble, France \\ CNRS, G-SCOP, F-38000 Grenoble, France \\ \{manel.brichni,lilia.gzara\}@g-scop.grenoble-inp.fr \\ ${ }^{3}$ STMicroelectronics, 850 rue Jean Monnet, Grenoble, France \\ \{manel.brichni,corinne.jeannet\}@ st.com
}

\begin{abstract}
Business Intelligence (BI) aims to support business users to make decisions by providing methods and tools to easily access and manage their information. Even if many organizations have employed BI applications, not all of them have been successful. Therefore, it is important to ensure that they fit business and users' needs in terms of information, knowledge and decision. In this paper, a proposal to apply BI for BI is elaborated. Analysing a BI system based on BI techniques is our main contribution and it is the core of our proposal. While involving users, our proposal aims to identify BI indicators and dimensions and to propose a system architecture allowing to analyse the current BI environment and make decisions about its use. A case study at STMicroelectronics has been conducted since it uses a BI system for making decisions.
\end{abstract}

Index Terms-Business Intelligence; Design science; Evaluation; Indicators

\section{INTRODUCTION}

Business Intelligence (BI) offers organizations potential for gaining insights in order to support decision making [1-3]. It ensures effectiveness in access to quality information from a variety of sources stored in different forms [4]. This is performed by means of collecting, storing and managing data [5]. BI applications and methodologies aim to support a deeper understanding of the business [5] and a provision of the appropriate data with the appropriate quality according to the users' needs [6].

Due to the evolution of business requirements and the availability of many BI tools, the evolution of BI is ever evolving [7]. This is the case of many organizations where BI acts on many business processes, uses different resources, involves different users' profiles and continuously generates information to make new business decisions. All of them are daily evolving. If they are not monitored, they will affect the BI functioning. In order to understand its activity, to ensure its performance and to take improvement actions, BI should be continuously evaluated and aligned with business objectives [8], [9].
The evaluation of BI applications is a topic widely discussed in literature [4], [5], [8-11]. It was evaluated from different points of view: technical, strategic, organizational or functional, etc. These studies provide several models to assess BI applications and make them achieve success. All these studies are mainly based on the definition of performance indicators according to defined success dimensions. They generally focus on the external environment of the system without considering its content, ie, used data, generated information or made decisions. Besides, most of these studies limited their work to an evaluation problem that we do not consider sufficient to analyse the BI system and make decisions about its use and improvement. This requires taking into account the system evolution perspective.

In this paper, in addition to the definition of performance indicators, we will expand the BI evaluation problem to the design of a full BI for BI system. Since it is the principle of $\mathrm{BI}$, our objective is to develop a BI system for evaluating, analysing and making decisions about the system itself. To this end, we propose the design of a global architecture of data warehouse for an industrial BI system.

Our solution is applied to STMicroelectronics currently using BI applications for their production activity.

The remainder of the paper is organized as follows. We present, firstly, the context of STMicroelectronics (section II), followed by our research questions and methodology (Section III) and related work (section IV). Next, the proposed solution for the development of a BI for BI system is presented in section $\mathrm{V}$. We end this paper with a conclusion and some perspectives (section VI).

\section{ThE CONTEXT AT STMicroelectronics}

STMicroelectronics is a global leader in semiconductors. It is the largest European company in its field. It existed since 1987. Our research is focused only at Crolles300 site 
in France, particularly the Manufacturing Solutions Group, having almost 75 employees and around 60 subcontractors. The main missions of this group are to support, maintain and adapt over time Crolles300 information systems to the level required by a world class $300 \mathrm{~mm} \_$Fabrication and R\&D plant (Research and Development). It aims as well to enrich Crolles300 information systems with the integration of new software modules needed by the latest semiconductor technologies. Our proposal is applied on one particular group, the "Reporting team" of the Business Intelligence activity. We describe its main activities and missions as well as issues related to our research problem, in the next section.

\section{A. Reporting team}

The activity of business Reporting consists in describing the organization activities through reports concerning one or more areas for a given period. Reporting aims to regularly inform those in charge of supervising activities or results. For example, by providing an overview of the time spent by one activity, a report permits to know which part of the manufacturing process is difficult to manage. It therefore identifies tasks that should be reviewed or controlled. It also puts under perspective the decisions by comparing activities evolution in short and long terms. In fact, to create a report, engineers have to define their objectives, to access to datasets, to select quality data, to define objects (indicators, dimensions, etc.), to create the report and to exploit it [12]. In the following, we will describe the BI system employed by the Reporting team.

\section{B. The Business Intelligence System for Reporting process at STMicroelectronics}

[13] defines BI as a broad category of technologies, applications, and processes for gathering, storing, accessing, and analysing data to help its users in making better decisions. In addition to these tasks, we consider that a BI system should promote the right exploitation of obtained results. Generally, results of BI are presented in the form of reports of different natures. To promote the right use and made decisions, such reports should be stored, shared and documented in order to be effectively reused. At STMicroelectronics, the BI is applied to the manufacturing activity, for example, to monitor the progress of wafers production and to target encountered problems during the activity.

To this end, in the Reporting team at STMicroelectronics, the BI system is composed of different tools interacting with each other, as shown in the Figure 1:

- A data warehouse is the core technical solution to design BI platforms. It is the collector of several and different data from various transactional information systems for analytical purpose [9], [14]. At STMicroelectronics, it treats manufacturing data about processes' evolution.
- Business Objects (BO) is the world's leading BI software company and it is owned by SAP ${ }^{1}$. BO allows accessing to data stored in rational databases, data warehouses and integrated applications (ERP, etc.) [15]. At STMicroelectronics, it is used for querying, reporting and monitoring data about the production activity.

- Safir Reporting portal is the platform for reports sharing. It allows the Reporting engineers at STMicroelectronics publishing created reports and allows users consulting them. It integrates some functionalities, for example, managing favourite reports, sharing them with colleagues or accessing to their documentation in Stiki, presented in the following.

- Stiki is a STMicroelectronics' wiki, designed and implemented in 2009 to cover the support, technical, business and project documentation. Currently, in the Reporting team, it is used as the main tool for knowledge sharing in the BI system as shown in our previous work [16]. We evaluated its use in order to identify areas of progress to accomplish its objectives for knowledge sharing.

- Blog Crolles300 is the notification platform used by the Reporting team to notify users by email for each new creation or update of a report. The email redirects them to a brief description of the report and its links to Safir and Stiki.

As described above and shown in Figure 1, the BI System of the Reporting team at STMicroelectronics is composed not only of BI tools but also of knowledge management tools ensuring an effective reports reuse while maintaining collaboration culture between users.

Actually, three different profiles interact between each other and with the BI system. As shown in Figure 2, users could be:

- A BI expert: he creates reports and maintains the BI system.

- A business expert: he provides the work methodology when a BI need is occurred and ensures good communication and use of the report.

- An end user: he asks for the creation of a report for his business needs.

As depicted in Figure 2, to create a report, an end user transmits his needs to a business expert (1). This one treats the request, defines requirements and transmits them to a BI expert (2). According to these requirements, BI expert creates the report, shares it in Safir portal, documents it in Stiki and notifies interested users via the Blog Crolles300 (3). Finally, the end user retrieves it (4).

In the organization, BI is facing several problems, mostly related to the evolution of business needs. Many BI tools are available. They are applied to many domains and business processes and continuously generating objects (reports or indicators for example) which can become obsolete or

\footnotetext{
${ }^{1}$ www.sap.com
} 


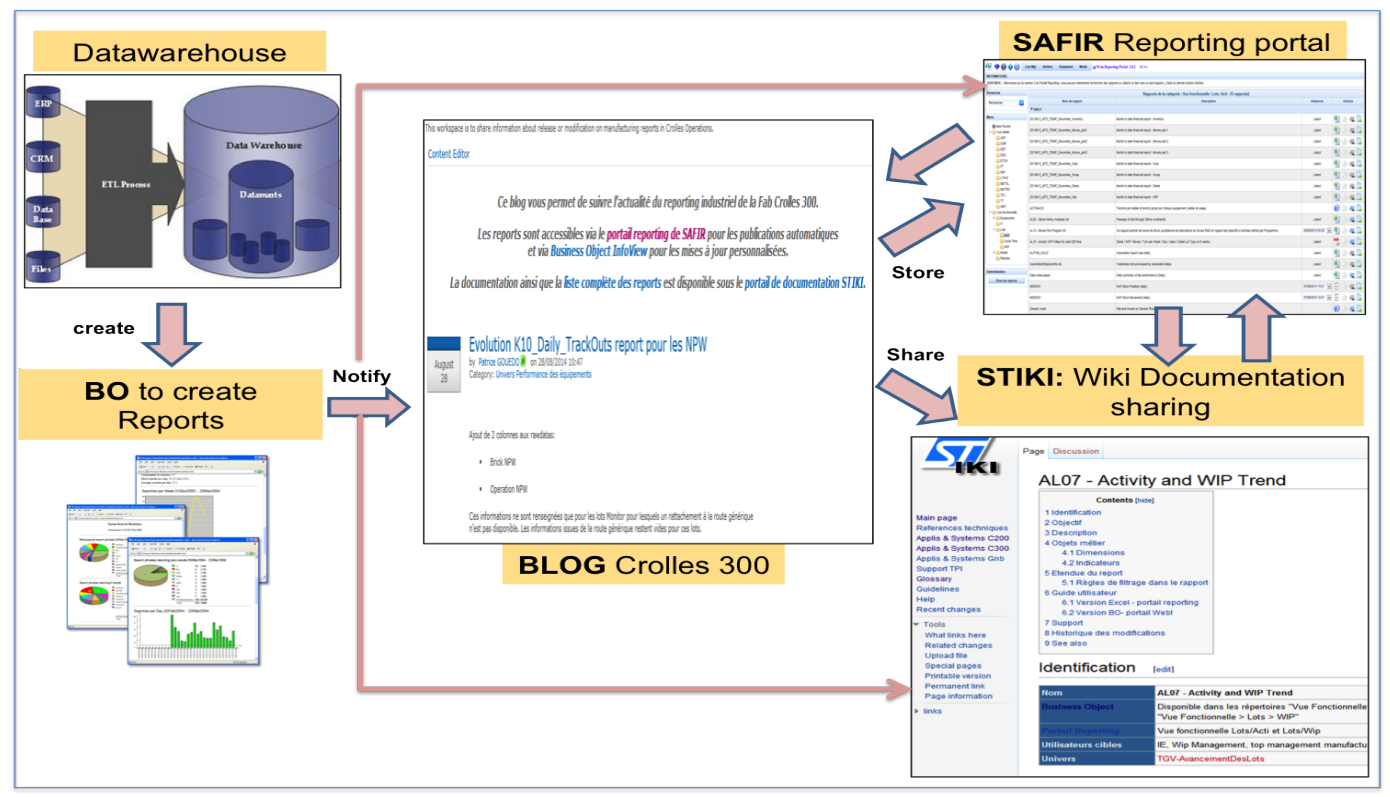

Fig. 1. The Business Intelligence System at STMicroelectronics

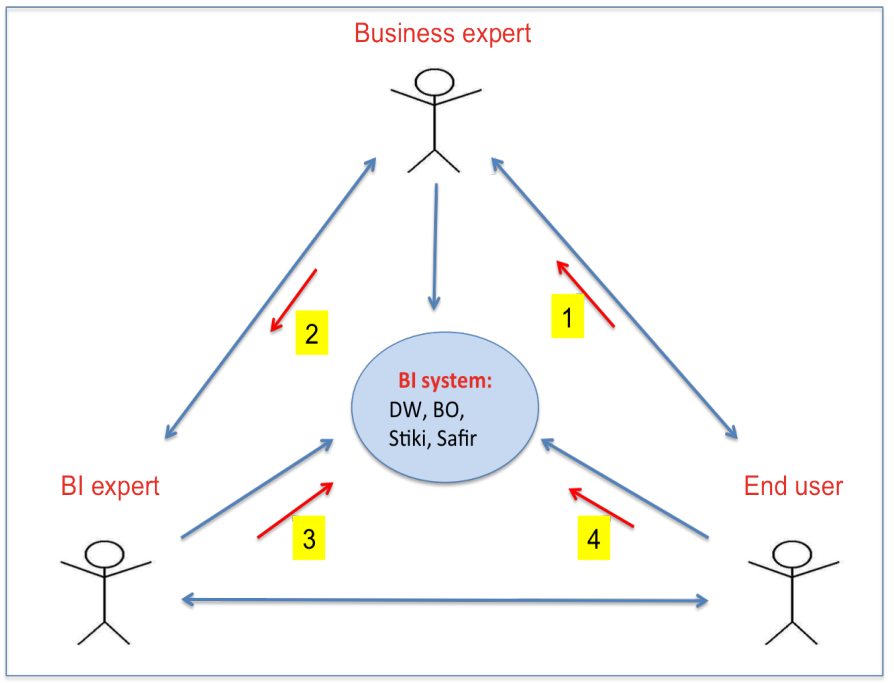

Fig. 2. BI users at STMicroelectronics

useless. Besides, its overloading of unused and duplicated ones may affect making the right decisions. This requires to align BI with business needs. However, currently, there is no solution allowing to monitor the BI system. This is the problem addressed in this paper based on the context at STMicroelectronics. We present in the following section our research questions as well as how to apply our research methodology.

\section{RESEARCh QUESTIONS AND METHODOLOGY}

\section{A. Research question and objectives}

Business needs are continuously evolving requiring the evolution of involved systems. As we have discussed above, to promote the performance of a BI system and take improvement actions, we think that it should be continuously evaluated and analysed while taking into account its use, evolution and maintaining after long time. Based on these findings, our main research question is:

- How to continuously align a BI system with business needs?

To answer this question, we aim to develop a solution for evaluating, analysing and making decisions about the BI activity and its evolution. Since these are the principles of BI, the developed solution will be a BI system applied to the system itself. To this end, our objectives are:

- To define the evaluation criteria in order to identify indicators for assessing BI system

- To measure and integrate them in the BI system

- To model a BI data warehouse with identified indicators and measures

However, such objectives could not be achieved without involving concerned users according to their uses, needs and generally, profiles. To this end, our research methodology, described in the following, is based on design science research and then integrates users thanks to a user centred approach.

\section{B. Research methodology}

A design science research seeks to create innovations that define ideas, practices, technical capabilities, and products. The purpose of the Design Science Research approach is at the intersection of people, organizations, and technology when designing an artefact that should impact and be impacted by people and their needs [17]. All of these characteristics lead us to adopt such a methodology.

As depicted in Fig. 3 [17], a design science research is composed of three cycles: Relevance, Design and Rigour: 
First, in the rigour cycle, design science draws from a vast knowledge base of theories and methods that ensures innovation. This knowledge could be based on the state of the art in the application domain of the research as well as on the existing artefacts and processes found in the application domain. Actually, we base on both of them. A literature review is done to identify and define $\mathrm{BI}$ evaluation criteria and their integration in the system (for example, we based on ISO 25000 model detailed in section IV). Besides, applying BI techniques to the BI system is our principle reusing existing design techniques and artefacts and ensuring innovation for our proposal.

Second, in the relevance cycle, good design science research often begins by identifying and representing opportunities and problems in an actual application environment (people, organizational systems, and technical systems). Therefore, we explored STMicroelectronics' current practices in BI, users' expectations and needs from $\mathrm{BI}$, advantages and limits of existing solutions of evaluation and desired improvements. To this end, we incorporate qualitative methods. To this end, face-to-face interviews are conducted and a list of participants is defined based on their profiles and activity on the BI system. Actually, the definition of characteristics and sub characteristics provided by ISO, indicators, measures and dimensions were identified. Interviews were conducted with the three different profiles of users presented in Figure 2, BI experts, business experts and end users of the BI system. Two users of each category participated. The objective was to involve users' experiences and expertise in the domain throughout our design process in order to collect their opinions and enrich our findings from different users' points of view. During the interviews, the procedure consisted, first, in presenting to users the definition of ISO characteristic and sub characteristic. Second, after understanding these definitions, for each one, they validated and enriched proposals of previous interviewees and they suggested indicators, measures and dimensions while justifying their opinion. Gathering users' suggestions helped to collect detailed information about the current BI activity and ensured a coherent solution. In a second stage, a questionnaire based on interviews results was proposed. It contains questions of two natures (open questions and leading questions). The questionnaire was mainly designed for BI users, to evaluate interviews results, particularly about the validity of identified indicators, measures and dimensions. In the questionnaire, for each identified indicator, the user is asked to evaluate corresponding measures and dimension according to rating scale, as well as to enrich the set of proposed measures. The questionnaire allows us to analyse the answers to see if users are generally satisfied with proposed indicators, measures and dimensions, so we are more able to validate our proposal. A summary of these experiments are presented in table I.

Third, in the design cycle, based on relevance and rigour,

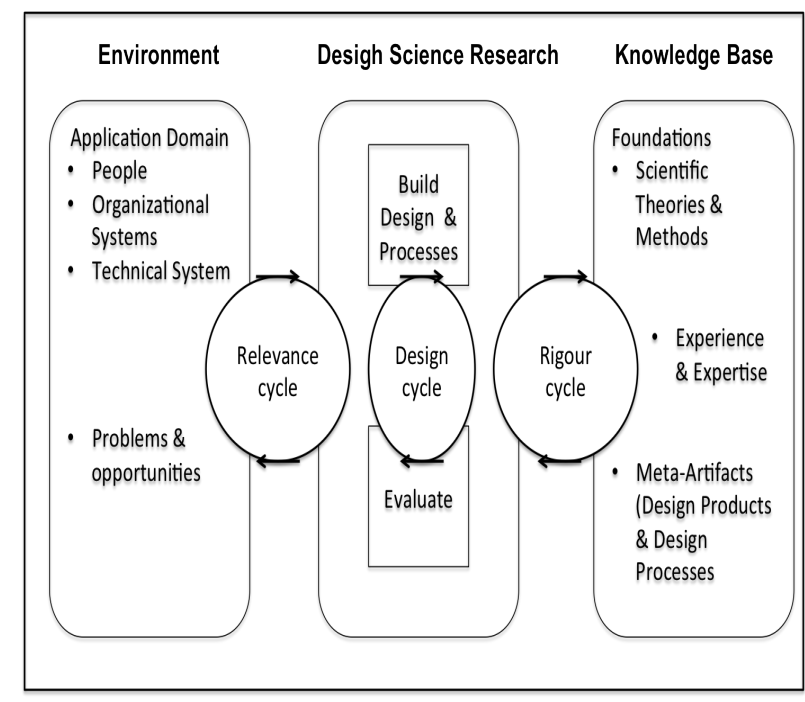

Fig. 3. Design Science Research

this cycle iterates between the construction of an artifact and its evaluation. In this step, based on the results of the literature review and the evolution of users' needs, evaluation criteria, indicators and measures are defined, tested and validated in order to be integrated later in the system.

As discussed in this section, the design science allows users to be integrated mainly in the cycles according to their uses, needs and profiles for the solution development. Following the steps described in Figure 3, it involves the three identified BI users' profiles in the study of the BI system. As a part of the rigour cycle, a literature review is presented in the next section in order to study how the problem of BI evolution is discussed.

\section{RELATED WORK: BI EVALUATION}

BI has been used in many sectors, for example, in magazine distribution [1], in banking [3] or in medical [18], [19]. However, since business needs regularly change and evolve, the problem of defining the right concepts and ensuring the right use of BI tools is very common. Many works have discussed the BI evolution problem [4], [5], [9-11], [20]. Two main categories of limits have been identified in literature case studies. First, they limited the BI evolution topic to an evaluation problem where most of works suggest evaluation criteria with almost no particularities to BI systems. The second limit concerns the proposed solution for BI evaluation.

\section{A. BI evaluation criteria}

In literature, case studies limited the evolution of BI topic to an evaluation problem. The evaluation, in general, has been much discussed in literature where the elaboration of standards such as ISO 25000 family for the development of software products through the specification of quality requirements 


\begin{tabular}{|l|l|l|l|l|}
\hline Methods & $\begin{array}{l}\text { Participants } \\
\text { profiles }\end{array}$ & $\begin{array}{l}\text { Number of par- } \\
\text { ticipants }\end{array}$ & Strengths \\
\hline $\begin{array}{l}\text { Face to face qualita- } \\
\text { tive interviews }\end{array}$ & $\begin{array}{l}\text { BI experts, busi- } \\
\text { ness users and } \\
\text { end users }\end{array}$ & $\begin{array}{l}6 \text { users where 2 } \\
\text { of each profile }\end{array}$ & $\begin{array}{l}\text { To gather in depth and detailed } \\
\text { information and feedbacks about } \\
\text { BI indicators, measures and dimen- } \\
\text { sions }\end{array}$ & $\begin{array}{l}\text { One hour per interviewee where } \\
\text { dimensions have been identified }\end{array}$ \\
\hline $\begin{array}{l}\text { Quantitative on-line } \\
\text { questionnaire to } \\
\text { measure the level of } \\
\text { agreement }\end{array}$ & $\begin{array}{l}\text { BI experts, busi- } \\
\text { ness users and } \\
\text { end users }\end{array}$ & $\begin{array}{l}20 \text { BI experts, } \\
\text { business users } \\
\text { and end users }\end{array}$ & $\begin{array}{l}\text { To quantify and assess the rele- } \\
\text { measures and dimensions }\end{array}$ & $\begin{array}{l}\text { More than half of users have } \\
\text { agreed with 40 measures }\end{array}$ \\
\hline
\end{tabular}

Table I. Experiments description

and evaluation quality characteristics. It provides a quality model allowing to decide which quality characteristics will be taken into account when evaluating the properties of a software product [21]. The product quality model defined in ISO 25000 comprises eight quality characteristics and their sub characteristics. The idea to base on formal specifications described in international standards is very interesting.

In addition, in literature, authors propose many BI evaluation models to elaborate their solution. They study the BI system from different points of view in order to elaborate a set of evaluation criteria. Basically, there are two main reasons for evaluating BI systems that guided authors to select their criteria.

The first reason is to help organizations to choose the most appropriate intelligent system to support their decision makers and to explore its strengths and weaknesses. In this category, we find works proposing criteria according to the software functional complexity [4], and functional suitability [11], and more general, according to its external and internal quality and quality in use [10]. For example, their criteria are defined for information delivery, BI integration and BI analysis points of view [4].

The second reason is to help organizations understanding how to achieve success with their BI systems. In this category, generally, BI evaluation criteria are represented with maturity models. For example, [9] integrates the following characteristics in his impact-oriented maturity model: BI deployment, BI usage, individual impact and organizational performance. For the same reason, [20] proposes a BI success dimensions affecting its use. He payes special attention to data integration, analytical capabilities, information content, access quality, the use of information in business processes and the analytical decision-making culture. In addition, [8] suggests that technological capabilities such as data quality, user access and the integration of BI with other systems are necessary for BI success.

In all cases, we note that authors efforts was focused on evaluating the BI system like any other tool. This means that we did not note particularities to BI systems. For example, they studied its organizational and technical environment as well as its quality and quality in use. Actually, the aim of BI is to offer users solutions to effectively make business decisions. This is the core of the BI activity. Therefore, analysing the system business content itself should be considered in the identified evaluation criteria, for example, analysing the use of business indicators, dimensions, reports, etc. Despite its importance, this point of view is not studied in literature.

\section{B. BI evaluation solution}

Second, more specifically to BI, literature suggested different solutions for BI evaluation. Most of them do not employ evaluation systems but used models or techniques. For example, [11] uses fuzzi TOPSIS technique, [22] uses BP Neural Network and [23] uses hierarchical Bayes model. Proposed models in literature are able to evaluate BI systems only to statically measure their qualities without helping to make decisions about their use and evolution. What BI systems need is a solution that continuously analyses its use while allowing making decisions about. To this end, our objective is to develop a BI system for evaluating, analysing and making decisions about the system itself, where our idea to use BI for BI.

In the following, we describe our proposal for applying BI for BI. It includes our design process based on design science, our purpose and solution specification.

\section{OUR PROPOSAL: BI FOR BI}

\section{A. The design process for our proposal}

We can not manage what we do not measure. It is an old management proverb that is accurate today and absolutely corresponds to the BI principle. This means that we can not ensure the right knowledge and decisions without the right data. As depicted in Figure 4, at STMicroelectronics, a production system generates data describing the evolution of the activity. A BI system bases on these data to measure, evaluate and analyse the production activity in order to generate knowledge and make decisions. Fundamental elements constitute the base of BI tools, for example, indicators, dimensions, reports, etc.

Since it is the principle of BI, our objective is to develop a BI system for evaluating, analysing and making decisions 


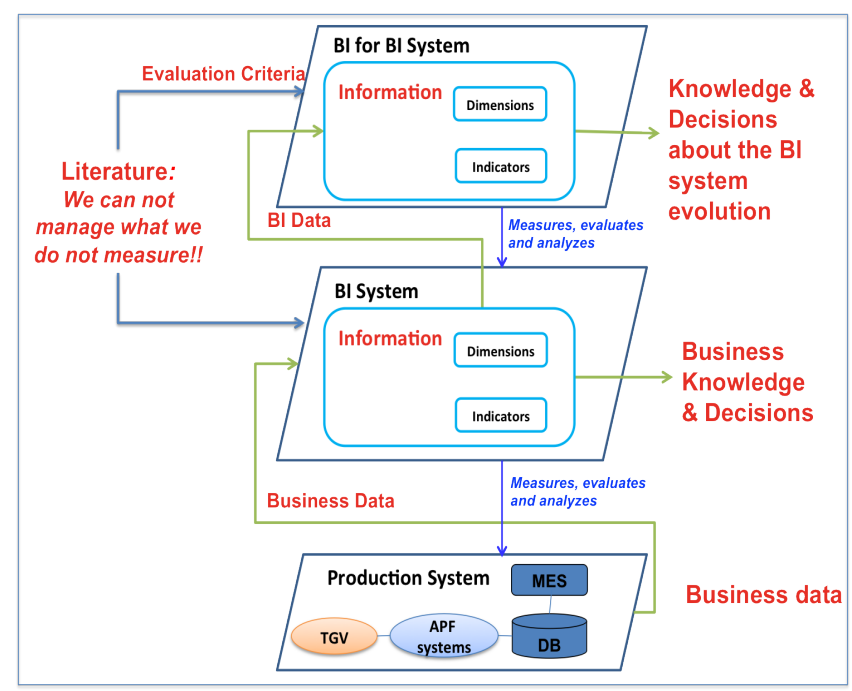

Fig. 4. BI for BI system

about the system itself, where the idea to apply BI for BI. As depicted in Figure 4, a BI for BI system applied to the current BI system consists therefore in using as well BI elements and techniques as follows:

- to define indicators and dimensions

- to define an architecture of the BI for BI system

- to model the dimensional data warehouse

The BI for BI system will be used to analyse the current BI system behaviour and to make decisions about its improvement actions. Monitoring the BI system will promote its activity, ensuring, consequently, making the right business decisions. For example, at STMicroelectronics, as part of a migration project, engineers have to migrate existing $\mathrm{BI}$ objects to a new data warehouse. First, they need to know which BI objects to migrate. To this end, analysing the uses and relationships between BI objects will help to decide which objects are useful and should be migrated. This will prevent the overloading of the new data warehouse with useless objects. Based on this principle, we detail in the following how we design our solution.

\section{B. Our solution specification}

To proceed, first, we propose a technical architecture for the design of the data warehouse of our BI for BI system. Next, for its design, BI modelling uses two main concepts, indicators and dimensions. It consists in modelling the relationships between them while providing an effective and simple representation of the activity at the lowest of granularity. This is the core of a BI modelling solution.

\section{1) Technical architecture:}

To build our system, a BI architecture plays an important role in organizing data, information and technology components that are used throughout the development and implementation decisions.
As depicted in figure 5, we choose a very classical BI architecture based on relational OLAP paradigm. The data components of the system architecture include the data sources, that in our case, correspond to BO, Safir Reporting portal, Stiki and the Blog databases. They include data describing each tool's activity. Next, by ETL -Extract, Transform and Load- processes, data from the four tools databases are extracted, transformed and loaded into the data warehouse, where data is stored in a suitable format for their decisional analysis. Oracle Data Integrater (ODI) is our ETL solution since it not only guaranties the highest level of performance but also the most cost-effective solution available today according to STMicroelectronics experience.

The target data warehouse is based on Oracle database as our object-relational database management system. We call it BI4BI DWR, where a multidimensional representation should help interactive analysis. To this end, OnLine Analytical Processing (OLAP) technique is used to represent data in a OLAP cube. Such a representation leads to conceptually straightforward operations to facilitate analysis. Typically, OLAP data is stored in star schema or snow flake schema. In our case, we use the snow flake schema to represent data in the data warehouse as described in the following. To effectively use data presented in the OLAP cube and generate information, different BI tools are used for reporting, analysis or data mining. BusinessObjects (BO) is our OLAP application solution for analysing, mining and reporting the BI activity, since it is used and known by the organization's users for its effectiveness.

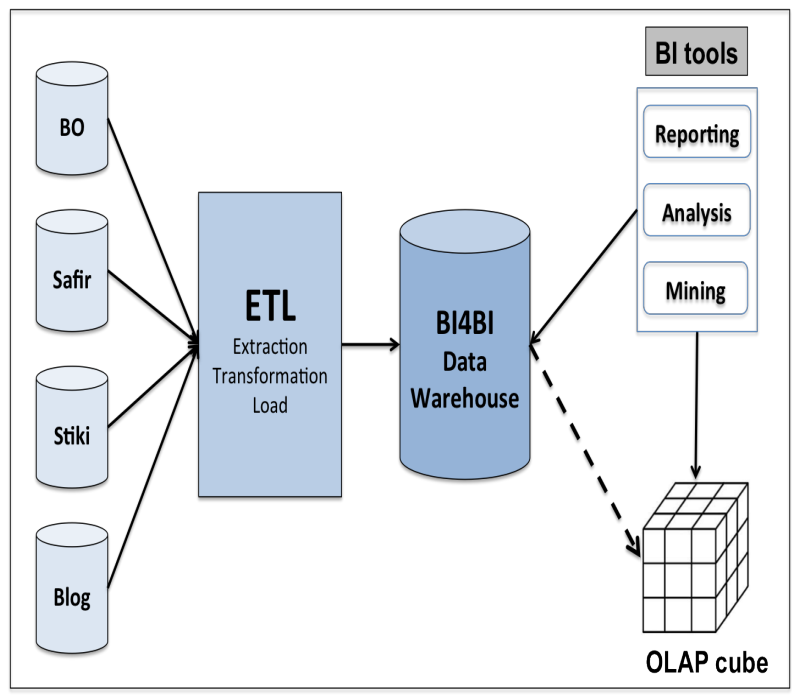

Fig. 5. The BI4BI data warehouse technical architecture

2) Indicators analysis and measures identification:

In BI, an indicator corresponds to the aggregation of data, generally quantitative, called measures. We note that we are searching for indicators to measure the BI activity. The particularity of our indicators is that are applied to the BI 
activity and its objects, for example, to the business indicators or dimensions. They may even reuse some existing ones, for example, a business indicator monitoring the production activity may be reused to monitor the reporting activity. We note that reusing BI techniques for BI systems is the core of our proposal.

In a first stage, we base on a literature review to identify evaluation criteria, as a part of the rigour cycle of the design science methodology. As we discussed in the state of the art (section IV), [10] based on ISO 25000 family to evaluate his BI system from three points of view: quality in use, external quality and internal quality. To base on formal specifications described in international standards is very interesting and this justifies our choice of the ISO model to select the evaluation criteria. ISO 25000 provides quality characteristics to evaluate the properties of a software product [21]. The product quality model defined in ISO 25000 comprises the eight quality characteristics and their sub characteristics, for example functional suitability, performance efficiency, compatibility or usability, etc. In order to measure the level of quality for each sub characteristic, appropriate indicators and measures should be assigned.

To construct STMicroelectronics' BI for BI system, among identified ISO characteristics, we first focus on the functional suitability and its sub characteristics in order to identify their associated indicators. Actually, despite the importance of the rest of characteristics, we classified them with users according to the BI evaluation's needs. We consider that the functional suitability is the characteristic that distinguishes the most the systems from each other, since it considers the degree to which the system provides specific functions that meet implied needs. the functional suitability is composed of the following sub characteristics:

- Functional completeness: Degree to which the set of functions covers all the specified tasks and user objectives

- Functional correctness: Degree to which a product or system provides the correct results with the needed degree of precision

- Functional appropriateness: Degree to which the functions facilitate the accomplishment of specified tasks and objectives.

To proceed, we use the definition of characteristics and sub characteristics provided by ISO in order to define indicators, their measures and later corresponding dimensions. Users' experiences and expertise in the domain at STMicroelectronics helped to enrich our findings from their points of view. As a result, our solution refers to various categories of indicators and measures. As shown in Figure 6, they concern:

- Involved BI users (Figure 2): for example, "the number of subscribed users" on the Blog to evaluate "its notification support" indicator

- Used BI objects: for example, "the number of duplicated or similar objects" in BO to measure "the objects cover-

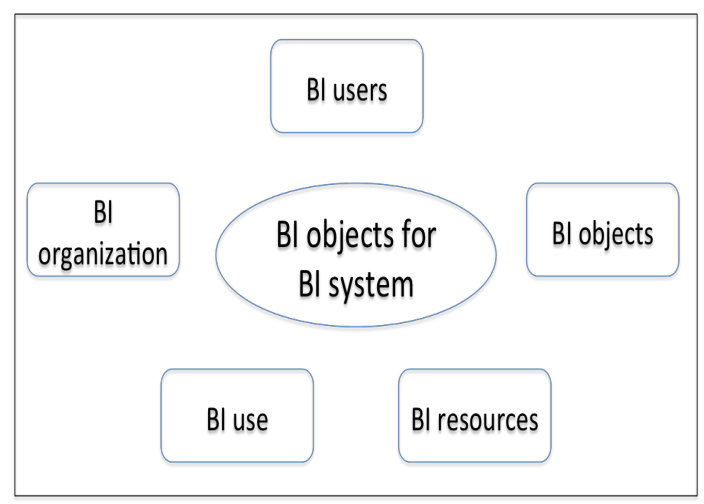

Fig. 6. Identified indicators categories

age" indicator

- Used BI resources: for example, "the number of distributed licences" of BO to measure "the resources uses" indicator

- Content BI uses: for example, "the number of contributions per Stiki page" to measure "the content uses" indicator

- Organization in the BI system: for example, "the ease of access to a report" measure to evaluate "the access to information" indicator

As a result, evaluation criteria, indicators and measures are defined for each tool. We present in this paper those corresponding to $\mathrm{BO}$ in table II. For example, to evaluate the functional completeness for $\mathrm{BO}$, we suggest to measure its objects coverage as well as its activity evolution. To this end, a set of measures is proposed for each one. To measure objects coverage, with users, we think that it is important to measure the number of $\mathrm{BI}$ objects uses, the number of covered domains, the number of duplicated BI objects, etc. Each measure could be subjective, based on the subjective judgement of a user, or objective, based on quantification where objective measures may vary according to a set of dimensions that we detail in the following.

\section{3) Dimensions identification:}

A dimension is an element constituting the context of an indicator. In BI, they are grouped into meaningful sets for users and decision makers. In $\mathrm{BI}$, dimensions represent business concepts and we often talk about a hierarchy of dimensions that could be geographical (cleanroom, town, etc), temporal (year, month, day, etc) or of products. For example, at STMicroelectronics, in the BI system we have as product dimensions: equipment, operation, lot, step, etc.

In our proposal, we do not talk about business dimensions but about dimensions to apply to identified measures analysing the BI activity (described in the previous section). To this end, with users, for each objective measure, we select appropriate dimensions. Our findings were validated with the questionnaire. As a result, we identified four sets of dimensions presented in figure 7 . 


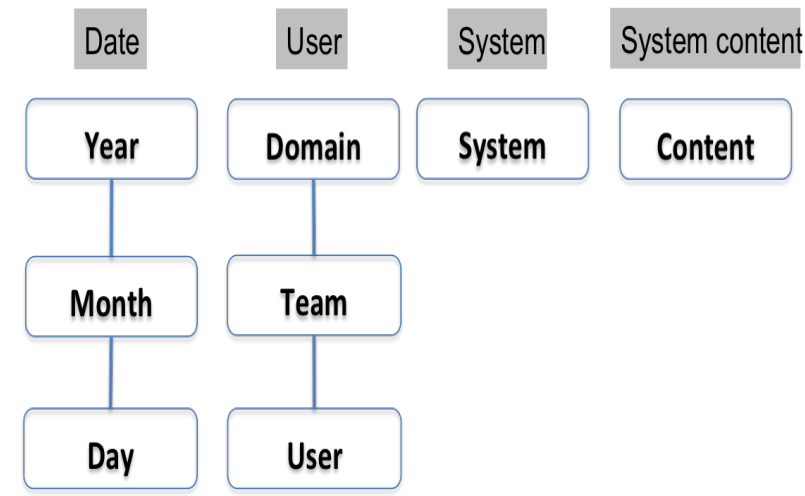

Fig. 7. BI dimensions

- Date dimension hierarchy: one or more time dimensions are often required. In BI, since we use objects to create reports and make decisions, the time dimensions could vary between the year to the day level.

- User dimension hierarchy: this hierarchy is composed of three levels:

- Domain dimension: BI system could be used for different domains within the organization. This is why, the domain is one dimension for some identified indicators. For example, at STMicroelectronics, domains could be: IT, finance, communication, etc.

- Team dimension: in a domain, the BI system could be used in different teams within the organization. This is why, the team is one dimension for some identified indicators. Each team belongs to one domain. This is why team is a sub dimension of domain. For example, in IT at STMicroelectronics, teams could be: local architecture, manufacturing execution systems, process control and automation, etc.

- User dimension: different users use the BI system in the organization. This is why several indicators are measured according to users. Each user works in one team. This explains why user is a sub dimension of team.

- System dimension: a system dimension could be any of STMicroelectronics' BI tools (BO, Safir Reporting Portal, Stiki or the Blog).

- System content dimension: for each system a content dimension is identified. For example, for BO, we identified its objects (Indicators, dimensions and reports). For Safir, indicators could be measured according to published reports or according to categories classifying these reports.

In order to understand the way dimensions are associated, in figure 8, we detail how the instances of the system dimension act with the instances of the system content dimension. As depicted in figure 8, a BI system could be BO, Safir, Stiki or the Blog. Each one has its own content type, where the content type of BO could be its objects: indicators,

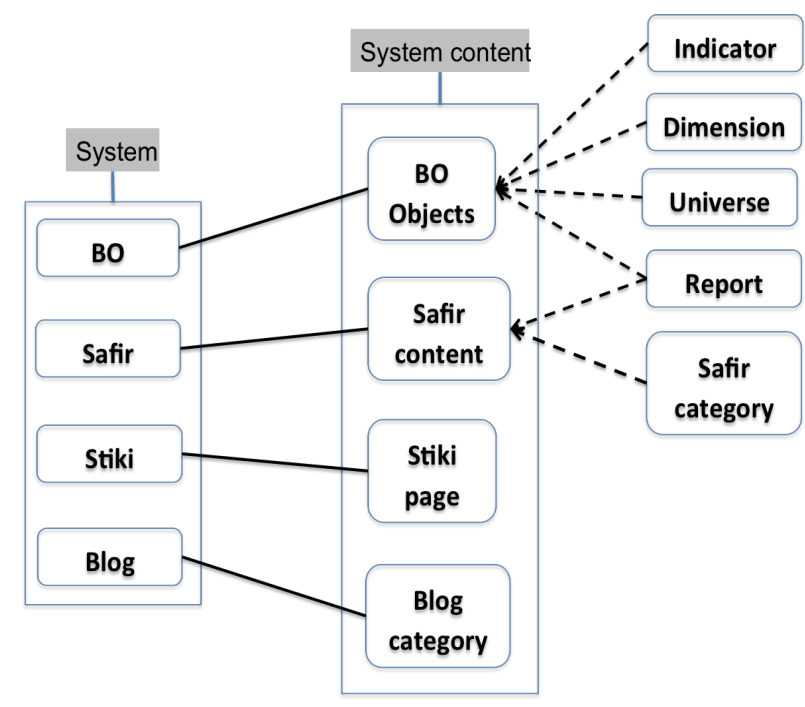

Fig. 8. Instances of BI dimensions

dimensions, universes or reports. The content type of Safir could be: reports or its categories.

In the table II, in addition to the definition of indicators and their measures, we associated to them the appropriate dimensions. For example, for BO, "the number of objects uses" measuring the "Object coverage" indicator is measured according to the following dimensions: time, domain, BO objects, user.

In this section, we presented BI dimensions that we apply to identified indicators (in section V-B2). Both of them will be used to model and develop the BI data warehouse.

\section{4) BI data warehouse modelling:}

In order to demonstrate how the BI could be applied to the BI system activity, in this section, we suggest an example of a BI data warehouse modelling based on identified indicators, measures and dimensions previously presented:

In the decisional solution section, BI experts would like to analyse their BI system activity and particularly the way objects are used in order to clean the system of unused objects. To this end, following objectives are identified:

- To track the BI objects' uses in BO

- To track the shared reports uses and users

- To track the documented reports uses and users

To address these issues, we elaborate the relationships between indicators and dimensions previously identified with a bus matrix. We present an example in table 9 where we determine the measures for the solution and decide which individual element to include with each dimension.

Based on these findings, in the following, we review the design of the dimensional model using the Snow Flake schema. 


\begin{tabular}{|c|c|c|c|c|c|c|c|c|c|}
\hline & Date & \multicolumn{3}{|c|}{ System } & \multicolumn{4}{|c|}{ Content } & \multirow{2}{*}{$\begin{array}{l}\text { User } \\
s^{e^{t}}\end{array}$} \\
\hline & 40 & $\beta^{O}$ & $s^{5}$ & skiv & 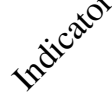 & $\theta^{x}$ & $e^{e e^{0}}$ & $s 0^{20}$ & \\
\hline The number of indicators & $\mathrm{X}$ & & & & & & $\mathrm{X}$ & & \\
\hline The number of dimensions & $\mathrm{X}$ & & & & & & $\mathrm{X}$ & & \\
\hline The number of created reports & $\mathrm{X}$ & & & & $\mathrm{X}$ & $\mathrm{X}$ & & & \\
\hline The number of accesses & $\mathrm{X}$ & & $\mathrm{X}$ & $\mathrm{X}$ & & & $\mathrm{X}$ & $\mathrm{X}$ & $\mathrm{X}$ \\
\hline The number of users & $\mathrm{X}$ & & $\mathrm{X}$ & $\mathrm{X}$ & & & $\mathrm{X}$ & $\mathrm{X}$ & \\
\hline
\end{tabular}

Fig. 9. Bus matrix

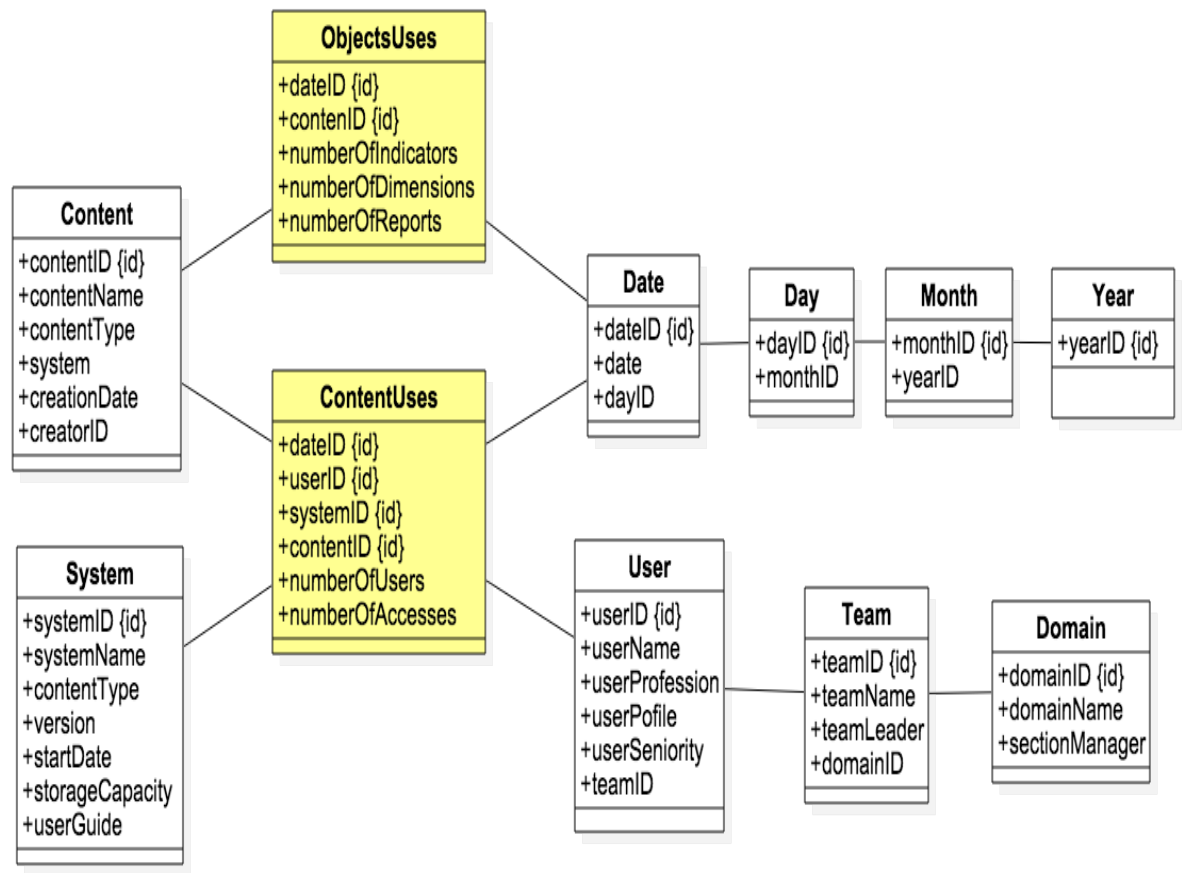

Fig. 10. Snow flake schema

In the snow flake schema, presented in figure 10, two types of tables are identified, fact tables (ObjectsUses and ContentUses) connected to dimension tables (Date, Month, System, Content, User). These tables are identified based on the bus matrix previously described in figure 9 , where we model, first, the fact table "ObjectsUses" used to track the BI objects' uses particularly indicators, dimensions for creating reports and the second one used to know if shared and documented reports are used or accessed, and by how many users. For the purpose of this article, the schema includes a simplified version of the problem with basic attributes in the tables.

For example, as a result of the development of the "ObjectsUses" table, we will be able to report:
- How many indicators and dimensions used per report

- In how many created reports, each indicator or dimension is used

Such findings will help engineers to determine the usefulness of the objects.

Up to now, we described how BI could be applied to BI based on its own techniques and particularly the data warehousing modelling.

\section{CONCLUSION AND PERSPECTIVES}

In this paper, we applied Business Intelligence for Business Intelligence at STMicroelectronics. We designed a BI system, evaluating and analysing the current BI system in order to help users making decisions about its use and 
evolution.

Particularly, we identified BI evaluation criteria based on findings in literature, we identified and validated with users a set of indicators, measures and dimensions. As a result, we designed the BI for BI system with its architecture and its data warehouse modelling.

In addition to the conceptual part of our proposal, we will develop a BI system with its objects, models and data warehouse to be applied to the current BI system. In the long term, identified indicators and measures will be evolved to make them platform independent, which is not discussed in this paper. To this end, we will make them independent of the specific system used to implement them. In that way, they will not be restricted to the type of used system. Such a generalized solution makes it applicable not only for monitoring a BI system but also information systems in general and in different contexts.

We note that throughout our proposal applying BI for BI, we aim to ensure a cycle of continuous improvement process. It would be one way to improve making decisions for systems and better target areas of progress.

\section{REFERENCES}

[1] N. McBride, "Business intelligence in magazine distribution," International Journal of Information Management, vol. 34, pp. $58-62$, 2014.

[2] J. Horkoff, A. Borgida, J. Mylopoulos, D. Barone, L. Jiang, E. Yu, and D. Amyot, "Making data meaningful: The business intelligence model and its formal semantics in description logics," in On the Move to Meaningful Internet Systems: OTM 2012. Springer Berlin Heidelberg, 2012, vol. 7566, pp. 700-717.

[3] S. Moro, P. Cortez, and P. Rita, "Business intelligence in banking: A literature analysis from 2002 to 2013 using text mining and latent dirichlet allocation," Expert Systems with Applications, vol. 42, pp. 1314 - 1324, 2015.

[4] C. Dell'Aquila, F. Di Tria, E. Lefons, and F. Tangorra, "Evaluating business intelligence platforms: a case study," in Proceedings of the 7th WSEAS International Conference on Artificial intelligence, knowledge engineering and data bases (AIKED'08), 2008, pp. 558-564.

[5] C. Jooste, J. Van Biljon, and J. Mentz, "Usability evaluation for business intelligence applications: A user support perspective," South African Computer Journal: SAICSIT 2013, vol. 53, pp. 32-44, 2014.

[6] J. Kaufmann and P. Chamoni, "Structuring collaborative business intelligence: A literature review," in System Sciences (HICSS), 2014 47th Hawaii International Conference on, 2014, pp. 3738-3747.

[7] I. Bill. (2014) Evolution of business intelligence. [Online]. Available: http://www.forestrimtech.com/evolution-of-business-intelligence

[8] Öykü Işık, M. C. Jones, and A. Sidorova, "Business intelligence success: The roles of $\{\mathrm{BI}\}$ capabilities and decision environments," Information \& Management, vol. 50, pp. 13 - 23, 2013.

[9] G. Lahrmann, F. Marx, R. Winter, and F. Wortmann, "Business intelligence maturity: Development and evaluation of a theoretical model," in System Sciences (HICSS), 2011 44th Hawaii International Conference on, 2011, pp. 1-10.

[10] J. Polańska and M. Zyznarski, "Elaboration of a method for comparison of business intelligence systems which support data mining process," $\mathrm{Ph} . \mathrm{D}$. dissertation, School of Engineering Blekinge Institute of Technology, 2009.

[11] S. Rouhani, M. Ghazanfari, and M. Jafari, "Evaluation model of business intelligence for enterprise systems using fuzzy \{TOPSIS\}," Expert Systems with Applications, vol. 39, pp. 3764 - 3771, 2012.

[12] H. Watson and B. H. Wixom, "The current state of business intelligence," Computer, pp. 96-99, 2007.
[13] B. Wixom and H. Watson, "The bi-based organization," International Journal of Business Intelligence Research, vol. 1, pp. 13-28, 2010.

[14] M. D. Sano, "Business intelligence as a service: A new approach to manage business processes in the cloud," WETICE Conference (WETICE), 2014 IEEE 23rd International, pp. 155-160, 2014.

[15] I. Hilgefort. (2011) Selecting the right sap businessobjects bi client product based on your business requirements for sap bw customers. [Online]. Available: http://www.scn.sap.com

[16] M. Brichni, N. Mandran, L. Gzara, S. Dupuy-Chessa, and D. Rozier, "Wiki for knowledge sharing, a user-centred evaluation approach: a case study at stmicroelectronics," Journal of Knowledge Management, vol. 18, pp. 1217-1232, 2014.

[17] A. R. Hevner and S. T. March, "Design Science in Information Systems Research," MIS Quarterly, pp. 75-105, 2004.

[18] N. Foshay and C. Kuziemsky, "Towards an implementation framework for business intelligence in healthcare," International Journal of Information Management, vol. 34, pp. 20 - 27, 2014.

[19] T. S. Cook and P. Nagy, "Business intelligence for the radiologist: Making your data work for you," Journal of the American College of Radiology, vol. 11,pp. 1238 - 1240, 2014.

[20] A. Popovič, R. Hackney, P. S. Coelho, and J. Jaklič, "Towards business intelligence systems success: Effects of maturity and culture on analytical decision making," Decision Support Systems, vol. 54, pp. 729 - 739, 2012.

[21] I. 25000. (2014) Iso 25000 software product quality. [Online]. Available: http://www.iso25000.com

[22] S.-L. Yan, Y. Wang, and J.-C. Liu, "Research on the comprehensive evaluation of business intelligence system based on $\{\mathrm{BP}\}$ neural network," Systems Engineering Procedia, vol. 4, pp. 275 - 281, 2012.

[23] N. Sano, K. Yada, and T. Suzuki, "Category evaluation method for business intelligence using a hierarchical bayes model," Cognitive Informatics Cognitive Computing (ICCI*CC), 2014 IEEE 13th International Conference on, pp. 400-407, 2014. 


\begin{tabular}{|c|c|c|c|}
\hline \multicolumn{4}{|c|}{ BO: Functional suitability criterion } \\
\hline \multicolumn{4}{|c|}{ Sub-criterion: Functional completeness } \\
\hline Indicators & Measures & Types of measures & Dimensions \\
\hline \multirow{4}{*}{ Objects coverage } & Number of BI objects uses & Objective & time, domain, BO objects, user \\
\hline & Number of covered domains by BI in the organization & Objective & domain \\
\hline & Number of available reports in $\mathrm{BO}$ & Objective & time \\
\hline & Number of duplicated or similar BI objects & Objective & domain, BO objects \\
\hline \multirow{5}{*}{ Activity evolution } & Number of reports created after BI modelling & Objective & time, domain, report, user \\
\hline & Number of requests for creating objects & Objective & time, domain, $\mathrm{BO}$ objects, user \\
\hline & Cycle time of reports creation & Objective & time, domain, report, user \\
\hline & Number of requests for correcting BI objects & Objective & time, domain, BO objects, user \\
\hline & Number of participants in creating a report & Objective & time, domain, report, user \\
\hline \multicolumn{4}{|c|}{ Sub-criterion: Functional correctness } \\
\hline Indicators & Measures & Types of measures & Dimensions \\
\hline \multirow{3}{*}{ Objects relevance } & The adequacy of BI objects to users' needs & Subjective & \\
\hline & Number of changes after the BI modelling & Objective & time, domain, $\mathrm{BO}$ objects \\
\hline & Number of different BO objects providing the same result & Objective & time, BO objects \\
\hline \multirow{3}{*}{ Objects uses } & $\begin{array}{l}\text { number of BI objects (indicators and dimensions) uses in } \\
\text { personal reports }\end{array}$ & Objective & time, domain, $\mathrm{BO}$ objects, user \\
\hline & $\begin{array}{l}\text { Number of reports uses (in public and personal folders } \\
\text { in BO) }\end{array}$ & Objective & time, domain, report, user \\
\hline & $\begin{array}{l}\text { The number of unused instances of reports running on } \\
\text { BO }\end{array}$ & Objective & time, domain, report, user \\
\hline Objects correctness & The accuracy of BO objects results & Subjective & \\
\hline Objects availability & Number of available BI objects at the scheduled time & Objective & time, domain, $\mathrm{BO}$ objects \\
\hline \multirow[t]{2}{*}{ Objects freshness } & $\begin{array}{l}\text { Number of available objects updated at the required } \\
\text { business time }\end{array}$ & Objective & time, domain, $\mathrm{BO}$ objects \\
\hline & The number of incidents for reports problems & Objective & time, report, domain \\
\hline \multicolumn{4}{|c|}{ Sub-criterion: Functional appropriateness } \\
\hline Indicators & Measures & Types of measures & Dimensions \\
\hline \multirow{4}{*}{ Objects manipulation } & Objects identification & subjective & \\
\hline & Objects organization & Subjective & \\
\hline & Objects exploitation & Subjective & \\
\hline & Objects documentation & Subjective & \\
\hline Exporting format & Graphics rendering & Subjective & \\
\hline \multirow{4}{*}{ Resources uses } & Number of trained people & Objective & time, domain \\
\hline & Number of distributed licences & Objective & time, domain \\
\hline & $\begin{array}{l}\text { Number of licences used for consultation and for contri- } \\
\text { bution }\end{array}$ & Objective & time, domain, BO objects, user \\
\hline & number of connections to $\mathrm{BO}$ & Objective & time, users \\
\hline
\end{tabular}

Table II. BO indicators, measures and dimensions 